\title{
Tissue Scurvy - not shaken baby
}

\author{
Michael D. Innis \\ Retired Haematologist, Princess Alexandra Hospital, Brisbane, Australia
}

E mail address:

micinnis@bigpond.com

\section{To cite this article:}

Michael D. Innis. Tissue Scurvy - Not Shaken Baby. Clinical Medicine Research. Vol. 3, No. 6, 2014, pp. 162-165. doi: $10.11648 /$ j.cmr.20140306.11

\begin{abstract}
Parents and carers are often falsely accused of injuring a child asserting that unexplained bruises, fractures, retinal and subdural hemorrhages with ischemic encephalopathy, the so called "Triad", are signs of physical abuse. However these features can be the result of Tissue Scurvy, a disorder of Vitamin C metabolism in which Vitamin C, although abundant in the plasma, is unable to enter the cell because of a lack of Insulin which is necessary to conduct the transfer of the Vitamin C into the cell. The cause is an autoimmune reaction to a viral, bacterial or parasitic infection or a vaccine which destroys the Insulinproducing Beta cells of the Pancreas, as evidenced by Hyperglycaemia and Glycosuria, in some genetically susceptible children. Here it is shown that the evidence of the Prosecution Experts alleging death from the Shaken Baby Syndrome was demonstrably flawed. The diagnosis Shaken Baby Syndrome is false and an innocent man was incarcerated. This irrational diagnosis should beabandoned.
\end{abstract}

Keywords: Autoimmunity, Tissue Scurvy, Triad, Non-Accidental Injury, Shaken Baby Syndrome

\section{Introduction}

Darryl Elliot was convicted for the murder of Amelia Bowmar on the evidence of doctors who alleged Amelia was Shaken to death. The basis of this allegation was the presence of a subdural hematoma, swelling of the brain with ischemic changes and hemorrhagic contusions in the cortex of the right and left superior frontal gyri of the brain. The brain and spinal cord showed evidence of old and recent injury which was attributed to "Non-accidental Injury".

The situation in which the carers of the child could offer no explanation for fractures, bruises, retinal and cerebral hemorrhages with ischemic encephalopathy was given the name "Shaken Baby Syndrome" in 1971 by a neurosurgeon Dr Guthkelch, who unsurprisingly, could offer no other explanation for the lesions in these children and incredible as it may seem nor could the doctors in the UK who reported on Amelia Bowmar a year ago.

Here it is shown that Amelia had a disorder of the coagulation system as evidenced by a raised INR of 1.3,(NR 0.89 - 1.2) an APTT of 39.6.(NR 25-35 sec) which would explain the brain and other hemorrhages.

However the evidence was ignored by the doctors alleging murder. Their preoccupation with the Shaken Baby Syndrome hypothesis also ignored the elevation of the level of Glucose in the Blood and the presence of Glucose in the
Urine of Amelia. Both these features are manifestations of an autoimmune response to antigenic stimulation as I have explained [1]. In this case it was, most probably, the vaccines given to Amelia a few days prior to her falling ill which initiated the process and Darryl Elliot was blamed.

Until the Medical Profession realizes that the Shaken Baby Syndrome is a fabricated diagnosis without a shred of scientific evidence, they are going to continue to falsely accuse innocent people.

Justice MacDuff sentencing Darryl Elliott said, "You have been convicted, on overwhelming evidence, of the murder of Amelia Bowmar, a little girl of 14 months whose care had been entrusted to you by your partner; her mother. Your job was to look after and protect her but, instead, she died at your hands".

The Judge was obviously completely convinced by the evidence of the doctors and the "overwhelming evidence" he refers to when analyzed is demonstrably "overwhelming incorrect" as demonstrated here. Each "expert's" evidence is examined separately. 


\section{Cases}

\subsection{Evidence of Dr P H T Cartlidge, Specialist Field Paediatrics}

Dr P H T Cartlidge in his evidence said, "in my opinion the head injury was not caused by a fall from the seat of the settee and I struggle to conceive how Amelia could have climbed higher". He goes on to say "the mechanism of nonaccidental head injury causing an acute encephalopathic illness, acute subdural bleeding and retinal haemorrhages is thought to be shaking, an impact or a combination of both. In shaking a child is often grasped around the chest and shaken.Squeezing the chest impairs the return of blood to the heart and thereby causes an increase in venous blood pressure. This in turn causes engorgement of the veins that traverse the subdural space on the surface of the brain. Shaking the child and/or hitting the child's head on a firm object leads to marked accellaration-decellaration forces that rupture these engorged veins, resulting in subdural haemorrhage The same shearing forces generated by shaking-impact can damage the parenchyma of the brain and also the spinal cord and cord roots".

\section{Comment}

Dr Cartlidge gives an accurate account of the mainstream opinion of the Shaken Baby Syndrome which is hypothetical without a shred of evidence and ignores crucial biochemical evidence such as Increased Glucose in the Blood (Hyperglycaemia), Abnormal Liver Function Test, Abnormal Coagulation Tests and Glycosuria (sugar in the urine) proving an Autoimmune Disease - TISSUE SCURVY [1].

\subsection{Evidence of Dr S J Hamilton, Forensic Pathology Unit Leeds}

Dr S J Hamilton, Forensic Pathology Unit Leeds who, with his colleague Dr Malcomson, performed the Postmortem examination on Amelia also gave evidence for the prosecution in which he stated "having considered all the findings with respect to the death of Amelia, it is my opinion that she as(sic) died of a head and spinal injuries. Neuropathology has revealed recent injuries including subdural bleeding, axonal injury in the medulla and spinal nerve roots, and fresh bleeding within older contusions within the brain itself. There is also evidence of older injury including older subdural bleeding and cerebral contusions. These are indicative of at least one, but possibly more than one, episode of significant head trauma. These neuropathological appearances are entirely consistent with non-accidental injury also referred to as paediatric head injury or sometimes "Adult Induced Injury". Although some authors have proposed theoretical models that would indicate a natural single underlying cause leading to the finding of subdural haematoma brain swelling and retinal haemorrhage to my knowledge these theoretical models have not been accepted by the Courts. The presence of this combination of findings is entirely consistent with a traumatic aetiology. The lack of external injury means that it is not possible to state that there has been an impact to the head, although the possibility of an impact that has not left an external injury must exist".

\section{Comment}

I am one of the authors that "have proposed theoretical models that would indicate a natural single underlying cause leading to the finding of subdural haematoma brain swelling and retinal haemorrhage" referred to by Dr S J Hamilton. Amelia had an elevated Blood Glucose and Glucose in her Urine, features which confirm a diagnosis of Tissue Scurvy [1].

\subsection{Dr Jayamohan Jayaratnam, Consultant Paediatric Neurosurgeon}

Dr Jayamohan Jayaratnam discussed all the available evidence including the fact that Initial blood clotting tests show some raised clotting times with an INR of 1.3, an APTT of 39.6 and an APTT ratio of 1.3.

With support from the evidence of Dr AL SARRAG he concluded "in my view this (is) sufficient support for my already formed view that there is likely to have been a traumatic explanation for Amelia's collapse. In view the globalized findings of ischemia, it was hard to place exactly what may have occurred. However with the focal injuries described by Dr AL SARRAG, in view of the absence of external signs of injury and in view of the bilateral severe ophthalmology findings, I believe it is highly indicative that a non-impact traumatic injury otherwise known as a shaking type injury has occurred to Amelia”.

\section{Comment}

Dr Jayamohan clearly identifies an abnormality of the clotting mechanism and then, for no reason whatever, chooses to ignore it as a cause of the bleeding in the skull and elsewhere. His illogical conclusion needs to be further investigated by his governing body for the safety of others with similar coagulation profiles.

\subsection{Dr Neil Gordon Stoodley, Consultant Neuroradiologist}

Dr Neil Gordon Stoodley Consultant Neuroradiologist concluded his assessment of the situation with the remark "I cannot think of a biologically plausible mechanism that would potentially directly link any event occurring toward the end of May 2012 and Amelia's presentation with a severe (indeed fatal)" encephalopathic illness at the end of July".

He continues:

"The scan abnormalities are all explicable on the basis of being due to an episode of non-accidental head injury. The mechanism of the injury is likely to have involved shaking. Majority medical opinion is of the view that what is likely to be required to produce such injuries is the backwards and forward movement of the unsupported infant head pivoting on the neck. It is believed that this leads to a degree of acceleration/deceleration and rotational forces and that the consequent differential rotation of the brain and skull leads to stretching of the 
subdural veins which cross the subdural space and it is this which leads to bleeding in the subdural space".

\section{Comment}

Dr Stoodley could not "think of a biologically plausible mechanism that would potentially directly link any event occurring toward the end of May 2012 and Amelia's presentation with a severe (indeed fatal)" encephalopathic illness at the end of July"

The biologically plausible mechanism Dr Stoodley has difficulty in thinking of that would potentially directly link an event occurring toward the end of May 2012 and Amelia's presentation with severe (indeed fatal) encepalopathic illness at the end of July is the MMR vaccine administered on $25^{\text {th }}$ May 2012.

An account of the condition-TISSUE SCURVY- which doctors including Dr Stoodley are obviously unaware of has been reported [1]. When doctors understand Tissue Scurvy can be induced by vaccines in genetically susceptible children and causes the signs and symptoms found in Amelia, they will stop diagnosing Shaken Baby Syndrome.

\subsection{Dr AL-SARRAJ, Consultant Neuropathologist}

Dr AL-SARRAJ provides a detailed account of the findings and concludes "the clinical history indicates presence of extensive bilateral retinal haemorrhages. These injuries, in addition to the presence of subdural haematoma and generalized ischemia (ischaemic encephalopathy) form the triad frequently seen in patients with non-accidental head injury. This is further supported by presence of subdural haematoma in the spinal cord, axonal injury in the pyramids of the medulla and axonal injury in the spinal nerve roots.

The presence of axonal injury in the pyramids and spinal nerve roots suggest head and spinal cord injury resulting from violent hyper-extension and hyper-flexion of the head and neck similar to those seen in shaking. The presence of extensive retinal haemorrhages supports this suggestion.

The pathological findings in the brain have to be considered closely with other findings of autopsy examination, radiological examination, eye examination and other investigations, before final conclusion. I will review the pathological findings in the brain and my interpretation if further information becomes available".

\section{Conclusion}

Head and spinal cord injury consistent with non-accidental injury.

\section{Comment}

There is no conflict with Dr AL-SARRAJ'S findings but it is the interpretation of those findings which are disputed. He is evidently prepared to review his interpretation if further information becomes available and I draw his attention to the fact that Amelia had Biochemical as well as Anatomical lesions. The Biochemical lesions of Hyperglycaemia, Glycosuria, Coagulation Abnormality (INR 1,3, APTT 39.6) all suggest an Autoimmune Disorder -TISSUE SCURVY [1] which I am sure Dr AL-SARRAJ is willing to consider.

\subsection{Dr R E Bonshak, Consultant Ophthalmic Pathologist}

Dr Bonshakgives a detailed account of the pathological changes seen in the eye when exposed to infection and injury and concludes "non-traumatic injuries which might possibly cause this form and extent of pathology include severe coagulopathy (bleeding disorder) leukaemia (with coagulopathy) septicaemia(with coagulopathy) meningitis(with coagulopathy)and catastrophic intracranial bleeding. I am not aware that these conditions have been identified".

\section{Comment}

The Hospital Records and Pathology Report of Amelia clearly showed:

1. INR (International Normalized Ratio) 1.3 (Normal Range 2 - 4.5)

2. APTT(Accelerated Partial Thromboplastin Time) 39.6 sec(Normal 27-35 sec)

Having been a Haematologist in a Teaching Hospital I HAVE REPORTED ON MANY THOUSANDS OF HAEMATOLOGICAL RESULTS. These results indicate that a Coagulopathy is present due to the reduction in the Clotting factors XII, XI, IX, and VIII and spontaneous bleeding is inevitable. It also indicates that the Liver is damaged because these factors are formed in the Liver.

Dr Bonshak has the evidence of a coagulopathy he requires to conclude "non-traumatic injuries which might possibly cause this form and extent of pathology" but does not have the knowledge required to interpret the evidence.

\section{Conclusion}

Darryl Elliot has been found guilty of murder on the evidence of doctors not competent in interpreting the Haematological evidence which clearly indicated a Coagulopathy caused the death of Amelia Bowmar.

Prior to migrating to Australia I was one of the Pathologists working for the Coroner in The West Riding of Yorkshire.

I declare that I have not made any statement that I know to be untrue.

\section{Acknowledgements}

I wish to thank Ms Elizabeth Peel, Darryl Elliot's mother, for providing me with the information used in the preparation of this manuscript.

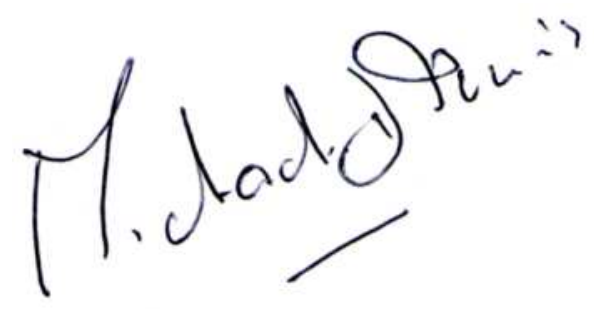

Michael D Innis MBBS; DTM\&H; FRCPA; FRCPath 


\section{Reference}

[1] Innis M D. Autoimmune Tissue Scurvy Misdiagnosed as Child Abuse, Clinical Medicine Research. Vol. 2, No. 6, 2013, pp. 154-157. doi: 10.11648/j.cmr.20130206.17

Darryl's letter sent to me from his prison cell

amelia had her mmr vaccine on may 23rd 2012, part from the nausea she experienced for a few days, how subtle would the vaccines have been in regards to any futher illness, 4 , if amelia collapsing was due to the reaction from the mmr vaccine, would my accident of dropping her heighten the risk to her.5, if amelia had any blood disorder that caused het to bruise easily, would ( in the words of the crown prosecution) my vigorous and violent shaking have brought on any external bruising to her upper torso,6, did my panic and subsequent shaking cause amelia more harm.7 I didnt mention to the hospital that I had shaken amelia in panic, if I had told them sooner, could it have save her life. 8 , medical experts did not believe my version of events, they dontbeleiveamelia could have entered into the condition I found her in, unless she was involved in some kind of severe head trama or nai, I beleive you have my version of the events, could you offer any explanation as to why ameliasconditon turned so rapidly. 9 amelias scan at grimsby showed no abnormalities to a trainee radiologist, however, at sheffield the next day a trained radiologist saw severe abnormalities, from 18.30 on the 29 th july to 10.30 on the 30 th, drsdidnt know about anything going on in amelias brain until a 2 nd scan was done at 13.30 due to amelias pupils being a different size, how much could have gone on in her condition between 18.30 and 13.30, that the drsdidnt see, would more seizures in this period of time have caused more damage to her brain, why was a trained radiologist not available in such a life threatening situation, 10, we was told they was checking for meningitis, diabetes, metabolic conditions, what else should have been explored? And if it wasnt explored, why wasnt it,11, the coagulation condition you found, how long did amelia have it, how would it or should it have been spotted, and how did it affect amelias condition, before and after she collapsed.12, what could have caused her levels of glucose to elevate and why wasn't it spotted.13, what did glucose in her urine represent, what effect would it have had on amelia,14, plunketts report is now 14yrs old, has there been any other short fall fatalities since then. Finally, was amelias death my fault,Thank you for taking so much time out to try and answer my questions, thank you also for helping me in my struggle to clear my name, I loved my daughter and never unlawfully harmed her in any way, not would I ever.myself and my family are truly grateful for the work you are doing for myself and amelias mum and family, they need the truth just as much as me, many thanks drinnis I am truly grateful. Darryl elliott, dated 12/03/2014" 